\title{
Behavioural and neurogenomic responses of host workers to social parasite invasion in a social insect
}

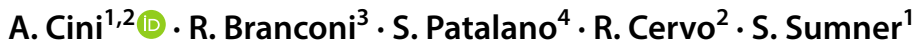

Received: 10 December 2019 / Revised: 7 March 2020 / Accepted: 20 April 2020 / Published online: 15 May 2020

(c) The Author(s) 2020

\begin{abstract}
The strong coevolutionary arms race between social parasites and their hosts has dramatically shaped the life-history traits of both parties. One of the main strategies exhibited by hosts in response to parasitism is reproduction by host workers. We lack a mechanistic understanding of how these defence strategies unfold and, specifically, whether hosts exhibit more subtle strategies to reduce the costs of parasitism from the outset. Here we test the hypothesis that there are both behavioural and neurogenomic signatures of worker responses to parasitism, prior to overt expression in the form of egg-laying; we test this using the social parasite-social host system of the paper wasps Polistes sulcifer-Polistes dominula. We characterized individual workers' position within the social interaction network of queenright and host colonies immediately after parasite usurpation, weeks before the workers' reproductive rebellion is evident. Parasitism influenced network centrality measures, with workers in parasitized colonies showing increased connectedness and centrality compared to those in unparasitized ones. Next, we quantified brain gene expression levels for five genes related to physiological and behavioural phenotypes in Polistes wasps. The gene Imaginal disc growth factor (Idgf4), thought to be responsive to changes in the social environment, was significantly down-regulated in workers from parasitized colonies; this may be an indication that parasitized workers are anticipating a shift toward a less worker-like phenotype in preparation for their reproductive rebellion. Our results provide the first evidence of early behavioural and neurogenomic responses of host workers toward the presence of an inquiline social parasite in a social insect.
\end{abstract}

Keywords Brood parasitism $\cdot$ Polistes sulcifer $\cdot$ Polistes dominula $\cdot$ Arms-race $\cdot$ Behavioural gene expression $\cdot$ Social network

Electronic supplementary material The online version of this article (https://doi.org/10.1007/s00040-020-00765-6) contains supplementary material, which is available to authorized users.

A. Cini

cini.ales@gmail.com

1 Centre for Biodiversity and Environment Research, University College London, Gower Street, London WC1E 6BT, UK

2 Dipartimento di Biologia, Università degli Studi di Firenze, Via Madonna del Piano, 6, 50019 Sesto Fiorentino, Italy

3 Department of Biology, Boston University, 5 Cummington Mall, Boston, MA 02215, USA

4 Institute of Basic Biomedical Sciences (IBBS), B.S.R.C "Alexander Fleming", Vari, Greece

\section{Introduction}

For social insect colonies, the threat of invasion by social parasites is part of life. From beetles to butterflies, many species have evolved the ability to exploit the socially acquired resources of insect colonies, e.g. their brood care, nest building and food supplies (Wilson 1971). Many of the social parasites that specialise in exploiting the social Hymenoptera (bees, wasps and ants) evolved from a social ancestor (Lowe et al. 2002): social parasites are widespread in ants (Hölldobler and Wilson 1990), allodapine bees (Smith et al. 2013), honeybees and bumblebees (Lhomme and Hines 2018) and wasps (Cervo 2006; Cini et al. 2019b). As parasites strive (in evolutionary terms) to be better exploiters of their social hosts, hosts respond by evolving strategies to reduce the chance and costs of invasion, leading to a coevolutionary arms race between the two species, and dramatically shaping their morphologies, behaviours and other 
life-history traits (Dawkins and Krebs 1979). Social parasite and host systems, therefore, present opportunities to study the ultimate and proximate bases of co-evolution.

Several lines of defence against the social parasite can be used by host species (Grüter et al. 2018). A first line of host defence is to avoid being conquered, for example through increased recognition or defensive strategies (Brandt et al. 2005; Ortolani and Cervo 2010): host species might evolve strategies that make it more difficult for the social parasite to integrate into the colony, for instance by evolving more complex chemical colony signatures which are more difficult to be mimicked by the social parasites (e.g. Bruschini et al. 2010; Lorenzi et al. 2014; Martin et al. 2011). In the event of an invasion, a second line of defence kicks in: host workers often launch a rebellion. Rarely can hosts expel their parasite; instead, they have evolved strategies to reduce the fitness of the parasite. Indeed, incomplete control by the social parasite over host worker reproduction has been highlighted in a few species of wasps and bumblebees (Cini et al. 2014; Reed and Akre 1983) and two main strategies have been reported. Workers may show an overt response, as seen in Temnothorax ants, where workers enslaved by the social parasite Protomognathus americanus kill up to two thirds of the parasite's pupae (Achenbach and Foitzik 2009). On the other hand, another common response is more covert, through worker reproduction (Cini et al. 2014). From the workers' point of view this strategy is clearly beneficial, as they can increase their direct fitness through the production of both males and females (as workers can mate and produce reproductive females (Strassmann et al. 2004)). By contrast, from the parasite's perspective, investment by workers in reproduction should be minimized in order to maximize worker investment in rearing the parasite's brood (Cini et al. 2014). Indeed, reproductive workers might be costly as they might contribute less to colony efficiency (e.g. Heinze 2008; Monnin et al. 2003).

However, both these strategies are 'last resorts' and only kick in several weeks after the parasite first invades. It seems remarkable that there has not been selection for host colonies to respond more immediately to the invasion of a parasite, as an early form of 'damage limitation'. Signatures of early response may be apparent through more subtle changes in behaviour and also in the expression of genes in the brain which are the front line response to changes in the environment (Robinson et al. 2005; Rubenstein and Hofmann 2015).

Here we explore whether there are signatures of early response to parasitism through subtle changes in behaviour and/or shifts in brain gene expression in a host paper wasp and its congeneric social parasite. Polistes sulcifer is an obligate inquiline (workerless) social parasite that invades nests of the social species Polistes dominula through violent fights (Cini et al. 2011; Turillazzi et al. 1990). The parasite replaces the host queen both behaviourally and chemically, achieving the top social rank in the dominance hierarchy (Cervo 2006; Cini et al. 2011; Dapporto et al. 2004). After usurpation, parasitized colonies produce parasite offspring, but also a small number of host offspring (Cervo 2006; Dapporto et al. 2004). Host workers are thus largely fooled (or suppressed) by the social parasite: they remain on the nest and rear parasite offspring, preferentially directing their provisioning effort toward the parasite brood as opposed to host brood (Cervo et al. 2004). However, recent results show that workers of parasitized colonies show signs of reproductive rebellion: they have more developed ovaries than workers from unparasitized colonies, and they lay eggs more rapidly when given the opportunity (Cini et al. 2014). However, this response is not evident until 6 weeks after parasitism (Cini et al. 2014), despite the fact that workers are able to develop their ovaries in only six days (Monnin et al. 2009) (Fig. 1a). The most parsimonious explanation, therefore, is that on usurpation host workers cannot detect the presence of the parasite, or if they can, the parasite is able to effectively suppress any ovary activation in host workers; but, after a considerable period of time (several weeks), the workers are able to overcome these issues and develop their ovaries. An outstanding question is whether workers show subtle signs of response to the parasite from the outset of usurpation, suggesting that perhaps they can detect and/or are imperfectly suppressed by the parasite; or whether workers are indeed effectively deceived/suppressed on usurpation, and remain so until the parasite is well established. Subtle signs of response to the parasite may be apparent in their behavioural social networks within the colony, and/or changes in brain gene expression (neurogenomic signatures), as the front-line response to changes in the social environment.

We tested these two hypotheses by comparing worker behaviour and brain gene expression in parasitized and non-parasitized $P$. dominula colonies across different stages of parasitism (Fig. 1). First, we characterized colony-level dominance networks in the early phase after parasite usurpation, within 2 weeks of usurpation, well before ovarian development is apparent (Cini et al. 2014) to determine whether there are any subtle changes in worker social behaviour as an early response to parasitism (Prediction 1). Dominance interactions are crucial to the regulation of worker activities in primitive insect societies (Naug 2008) and dominance interaction networks have been shown to reflect changes in social structure following major events such as changes in colony composition [e.g. queen removal, (Bhadra and Jordán 2013; Bhadra et al. 2009)]. Moreover, changes in individual network properties correlate with changes in behavioural phenotype in response to colony-level changes in social structure (Bhadra and Jordán 2013). We characterized the network positions of individual host workers using centrality measures that characterized both the local (first 


\begin{tabular}{|l|l|l|l|l|}
\hline PARASITE: Polistes sulcifer & $\begin{array}{c}\text { Parasitized workers } \\
\text { physiological reaction }\end{array}$ \\
physiological reaction
\end{tabular}

Fig. 1 A summary of the experimental design. (a) the model system, Polistes sulcifer (the social parasite, above) and Polistes dominula (the host, below) and the timeframe used in the experiment for sampling behavioural and gene expression patterns; (b) workflow and

order) and "extended" (second to n-order) networks for each individual (Pinter-Wollman et al. 2014; Wey et al. 2008); we then compared social network features in workers from parasitized and unparasitized colonies. If parasitism has an effect on workers' social behaviour soon after usurpation, we expect to find differences in the dominance network metrics of workers from parasitized and unparasitized colonies. Specifically, as dominance behaviour and reproductive skew are positively correlated in this species (Dapporto et al. 2010; Reeve et al. 1991), we predict workers from parasitized colonies to have higher centrality (i.e. engaging in more dominance interactions) than in unparasitized colonies (Fig. 1b).

To determine whether workers show any neurogenomic changes in response to the early stages of parasitism, we quantified brain gene expression for a set of five candidate genes in workers from parasitized and unparasitized colonies. We chose genes known to indicate response to changes in the social environment and reproductive status in Polistes (Cini et al. 2015; Ferreira et al. 2013; Manfredini et al. 2018; Sumner et al. 2006; Toth et al. 2014; Toth et al. 2007) (see Table 2 for details). If workers detect a change in their colony (putatively, the presence of the parasite herself) soon after usurpation, we expect the gene expression of workers in parasitized and unparasitized colonies to differ (Prediction 2). In particular, we expect to find some downregulation of worker-biased genes and upregulation of queen-biased genes in parasitized workers (Table 2), indicating an attempt to instigate a brain transcriptomic shift toward a reproductive phenotype (Fig. 1c). predictions for dominance network analysis; (c) workflow and predictions for brain gene expression analysis (image credits: Rebecca Branconi, Leonardo Platania)

\section{Materials and methods}

\section{Prediction 1: Positions of workers differ in social networks of parasitized and unparasitized colonies}

In Spring 2012, eight pre-emergence multiple foundresses colonies (range 2-4 foundresses) of P. dominula were collected in the vicinity of Florence (Tuscany, Italy). Each nest was fixed to the ceiling of a glass cage $(15 \mathrm{~cm} \times 15 \mathrm{~cm} \times 15 \mathrm{~cm})$ and maintained under controlled laboratory conditions with ad libitum sugar, water and paper material for nest building. All colony members and any newly emerged individual were individually marked with enamel paint dots (Testor Corporation) on the thorax and the wings before each observation. P. sulcifer females were collected in Spring from their overwintering sites at $2050 \mathrm{~m}$ altitude on Monti Sibillini (Umbria-Marche, Italy), transferred to the laboratory and kept in glass cages $(15 \mathrm{~cm} \times 15 \mathrm{~cm} \times 15 \mathrm{~cm})$ with ad libitum water and sugar, under controlled and constant low temperature inside a fridge, until it was time for their "activation" (temperature and exposure to direct natural and artificial sunlight following the protocol used by Ortolani et al. 2008). In the last week of May, when usurpation usually occurs in the field (Cervo and Turillazzi 1996; Ortolani et al. 2008; Turillazzi et al. 1990), four randomly chosen host colonies were subjected to parasitism by introducing a social parasite female inside the glass cage of a putative host nest (following the protocol in Cini et al. 2011) (Fig. 1a). Parasitism 
was considered successful only when the parasite exhibited typical behaviours of a dominant social parasite (i.e. stroking the abdomen on the nest cell rims and dominating host individuals (Turillazzi et al. 1990)). Parasitized and unparasitized colonies were reared under the same conditions for two weeks, until the end of the experiment. Unparasitized and parasitized colonies did not differ in nest size (number of cells) nor in colony size (number of adult colony members at the beginning of the experiment) (Mann Whitney test, nest size $U=4, p=0.314, n=4$; colony size: $U=7, p=0.881$, $n=4$; Unparasitized colonies; mean \pm st.dev: $54.80 \pm 3.95$ cells and $13.00 \pm 2.94$ adults; Parasitized colonies, mean \pm st. dev: $63.53 \pm 9.68$ cells and $10.60 \pm 2.22$ adults). All colonies appeared healthy, with brood of all stages in the comb at the start of the experiment.

Each colony $(n=8)$ was videotaped for $1 \mathrm{~h}$ between 10 am-3 pm (when wasps are most active) on the day before usurpation, on day 1 , day 7 and day 14 after usurpation (Fig. 1a); a total of $4 \mathrm{~h}$ of video observations were recorded for each of the four parasitized colonies and for each of the four unparasitized colonies.

All Ritualized Dominance Interactions (hereafter RDI) that occurred between any two individuals were recorded, noting the identities of the actor (i.e. the wasp that started the interaction) and the recipient. RDI includes domination acts, in which one wasp (the dominant individual) climbs on and antennates another wasp (the subordinate), and trophallaxis, i.e. liquid food exchange by mouth-to-mouth contact (Cini and Dapporto 2009; Pardi 1948) (Fig. 1b). Both interactions are directional, with dominant individuals (the actor) performing and not receiving RDI, and subordinate individuals receiving and not performing RDI. During trophallaxis the liquid is regurgitated by the subordinate individual toward the dominant one. To maintain the directionality of dominance, for trophallaxis the actor was considered the individual which received the liquid (i.e. the dominant individual) and the recipient the individual who gave the liquid (the subordinate one) [as in (Cini and Dapporto 2009)].

In animal social networks, usually each node represents an individual, and each link between two nodes ('edge') represents the existence and direction (from actor to recipient) of a dyadic interaction, and (in weighted networks) indicates the strength of the interaction (i.e. a value proportional to the frequency) by the thickness of the link. In the present study, we built directional weighted networks of parasitized and non-parasitized wasp colonies as video observations allowed us measuring the frequency of interactions, and RDI are directional interactions. Social networks for each colony at each time point (Fig. 1) were computed with UCINET 6 (v 6.665) (Borgatti et al. 2002).

For each individual and for each network we computed five standard node-centrality measures: weighted node degree, closeness centrality, eigenvector centrality, betweenness and clustering coefficient (Table 1). Tie direction is a critical aspect of a dominance network, as more dominant individuals deliver, but do not receive, RDIs, while the opposite is true for subordinates. For this reason, we separately computed Incoming (i.e. received) and Outgoing (i.e. performed) measures for the three directional network metrics weighted node degree, closeness centrality and eigenvector centrality. For example: outgoing weighted degree considers only the interactions started at a specific node and directed toward another node (e.g. by the actor of RDI); incoming closeness considers only the incoming ties (e.g. by the recipient of RDI).

Centrality measures are often positively correlated in social networks (Valente et al. 2008; Wey et al. 2008). For this reason, we applied a Principal Component Analysis (Varimax rotation) to reduce the number of variables into smaller number of uncorrelated principal components, extracting principal components with eigenvalues greater than 1 , and then using these as social network metrics in our study (see results section and Supplementary material

Table 1 The network metrics used in the study to describe worker position in the colonial social network

Network metrics. Considered and their definition (Krause et al. 2009; Wey et al. 2008)

Weighted node degree. Number of direct links (or 'edges') that a focal individual has with other individuals, weighted by the strength of each links (number of interactions)

Closeness centrality. Average distance (measured as the number of edges) from a given starting node to all other nodes in the network; this measure captures how well connected an individual is to all the other individuals in the nest

Eigenvector centrality. Assigns relative scores to all nodes in the network based on the concept that connections to high-scoring nodes (those with more edges) contribute more to the score of the node in question than equal connections to low-scoring nodes; this method captures the notion that a degree extends beyond just the first-order layer of connections that a focal node has

Betweenness centrality. Measures how often a node appears on shortest paths between nodes in the network. For every pair of vertices in a connected graph, there exists at least one path between the vertices such that the sum of the weights of the edges (for weighted graphs) is minimized and the betweenness centrality for each node is the number of these shortest paths that pass through the node

Clustering coefficient. Measures the density of a focal node neighborhood, i.e. the degree to which an individual's immediate neighbours are connected (Krause et al. 2009) 


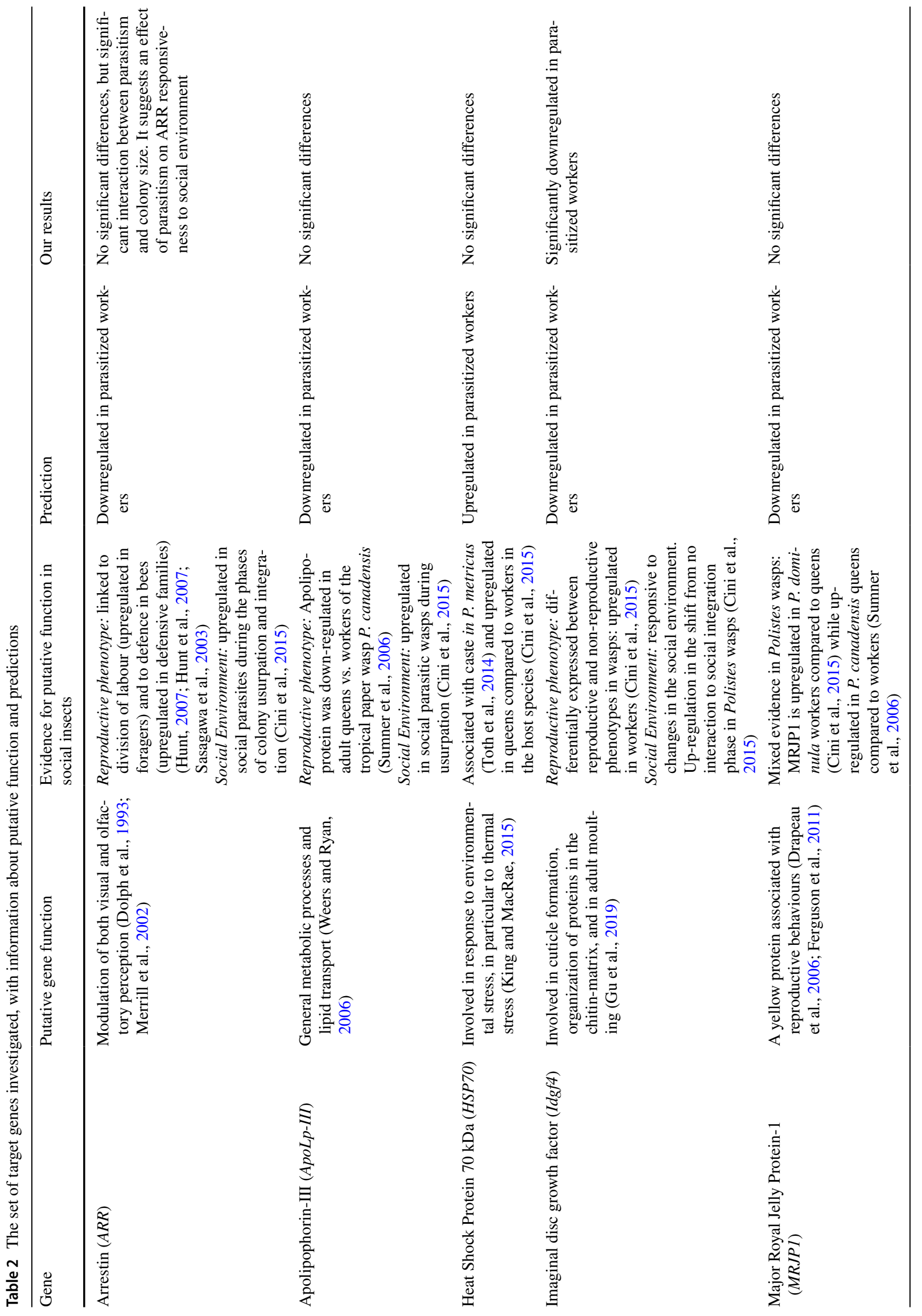


Dataset3.csv). Comparisons made with each single network measure gave an overall similar pattern (data not shown).

We assessed the importance of parasitism on individual centrality in the dominance network running three nodelevel regression analyses (implemented in Ucinet, using 10,000 random permutations and 32,767 random number seeds), one for each centrality measure: outgoing centrality $\mathrm{PC} 1$, incoming centrality PC2, and clustering coefficient PC3. We used each centrality measure as dependent variable, and parasitism status and colony of origin as independent variables.

\section{Prediction 2: Worker brain gene expression differs in parasitized and unparasitized colonies}

Data examined here come from samples collected during a previous experiment, which were reared in a similar way to the above behavioural experiments (Cini et al. 2015). Wasp samples were collected from Central Italy in June 2005. Unparasitized colonies were collected from the field and parasitized colonies were obtained either by field collection or laboratory usurpations (as above). Colonies were brought to the lab and reared under controlled laboratory conditions (as above). For each colony, behavioural observations confirmed that parasites were dominant and successfully took over the nest (Cini et al. 2011). Workers were collected within the first two weeks of usurpation. Forty-two workers were sampled, of which 26 were from 14 unparasitized colonies and 18 were from 9 parasitized colonies. Two workers were analysed per colony, except for two colonies in which only one worker was analysed. Wasps were killed directly into RNAlater (AMBION) and stored at $-20{ }^{\circ} \mathrm{C}$ until qPCR analyses (performed in 2009). Nest size (number of cells) did not differ between unparasitized and parasitized colonies (nest size, Mann Whitney $U=53.5, p=0.537$; $\mathrm{n}=14$ vs 9); colony size (i.e. number of colony members) was slightly larger in unparasitized colonies (Mann Whitney $U=29.5$, $p=0.036 ; \mathrm{n}=14$ vs 9 ), so we included colony size in the model to account for this (see below).

To select the genes to test this prediction, we initially referred to the only study published at the time, which identified several genes differently expressed between the workers and the queen of Polistes canadensis (Sumner et al. 2006). At the time, neither genome nor transcriptome data for $P$. dominula nor $P$. sulcifer were available; accordingly, we tested different primers specific to $P$. canadensis on synthesized cDNAs from our two species of interest. Since genes associated with worker behaviour appear to be conserved across species, using genes identified in a congeneric species like $P$. canadensis is an appropriate approach. A first selection of candidate genes was made on the basis of the amplification of a single band observed on agarose gel (Supplementary Material). Then we isolated, purified and sequenced each single band to verify that the amplified genes were indeed homologous between the Polistes species (Supplementary Material S2). Finally, we aligned all sequences and measured the percentage of identity using CLUSTALW and nucleotide BLAST bioinformatic tools (Supplementary Material S2). Five candidate genes Apolipophorin III, ApoLp-III; Arrestin, ARR; Heat shock protein 70KDa, HSP70; Imaginal disc growth factor, Idgf4; Major Royal Jelly Protein 1, MRJP1, (Table 2) were selected for further quantification of gene expression by q-PCR, using Tubulin alpha1 chain, TUBA $1 C$ as a control. On the basis of previous studies (Cini et al. 2015), four out of the five candidate genes were expected to be downregulated in workers from parasitized colonies compared to workers from unparasitized colonies (ARR, ApoLpIII, Idgf4, MRJPl) while one gene (HSP70) was expected to be upregulated (see Table 2).

RNA extraction from $P$. dominula and $P$. sulcifer and quantification of q-PCR product followed the protocol in (Cini et al. 2015). Briefly, total RNAs were isolated with TRIZOL (Invitrogen) from individual dissected brains and DNase treated (Turbo, PROMEGA) (Fig. 1c). Quantification was conducted using a Nanodrop and samples were checked for RNA degradation on an agarose gel. First strand cDNAs were synthesized with $1 \mu \mathrm{g}$ of total RNA and reverse transcribed with Retrotranscriptase kit (AppliedBiosystem). cDNAs were amplified by quantitative PCR using the LightCycler- DNA Master SYBR Green I kit (AB) and the following primers: HSP70-5'CTGTTCTTAGCGGCAATGGTC-3' and 5'- TGGACA AATCTACTGGCAAGGAG-3', Idgf4-5'-TGCCATACT TTTTCGATGGGT-3' and 5'-GCGTTCCACCGATAA TAGCTG- 3', TUBA1C-5'- AGCACCATCGAATCG TAAGGA-3' and 5'-ACATTCCGACTGCGCATTTAT- 3', ApoLp-III-5'-AGCACGAATTTCGCCAATAAA-3' and 5'-CGCATCTGGAATTTGAGCATT-3', MRJP1-5'-GCT AGACCAACGACACCATCATC-3' and 5'-TTCGCGAAA AATCCAAAAGC-3', ARR - 5'-TCGCAGTGTTGCACC ACAA-3' and 5'-TCACTTTCTCTCAGGGCAAACTT$3^{\prime}$. Since primer sets were chosen to target gene regions where both species share $>90 \%$ similarity (Supplementary Material S2), comparable amplification efficiencies were obtained in both species. Gene specific standard curves were established using serially diluted cDNAs from a mixture of both host and social parasite brain RNAs. Q-PCR was performed on a LightCycler STEPONE (AB). Three technical replicates were run for both samples and standards. Cycle Threshold (CT) values were removed if amplification baseline was $15<$ or $>35$ and if melting curve patterns were not sigmoidal. Selected CT were submitted for quality control using Q-base software, and normalized against tubulin. Absolute quantitative means were derived from triplicate CT values obtained from the standard curve, normalized to the value of the 
tubulin gene. Stability of tubulin gene was validated using GeNorm software. Primers' specificity for each gene was checked by RT-PCR (PCR masterMix, PROMEGA) on both parasite and host cDNAs. Unique band amplification was confirmed on an agarose gel, after which PCR products were purified (purification kit, QUIAGEN), sequenced (BigDye kit, $\mathrm{AB}$ ) and homology between parasite and host sequences was checked with CLUSTAL-W. After data quality control, sample sizes of workers from unparasitized and parasitized colonies for each of the five genes (see below and Table 2) were as follows: ApoLp-III: 26 vs 18 ; ARR: 11 vs 11 ; HSP70: 26 vs 18 ; Idgf4: 26 vs 18; MRJP1: 12 vs 12 .

To account for the non-independence of data (i.e. workers belonging to the same colony), we used a generalized estimating equations (GEE) approach, which extends the generalized linear model to allow for analysis of correlated observations, as is the case for clustered data (Burton et al. 1998). This approach is robust against misspecification of the error structure model and has relaxed assumptions on data distributions (Hubbard et al. 2010; Overall and Tonidandel 2004). We selected the best model on the basis of the "quasilikelihood under the independence model" criterion QIC, by choosing the model parameters that resulted in the smallest QIC (Pan and Wall 2002). We ran a GEE for each gene, with the following parameters: tweedie probability distribution, log link function, independent working matrix correlation. We ran a full factorial model which tested the effect of parasitism (fixed effect), colony size (covariate) and their interaction, by controlling for colony of origin (included as the subject variable). GEE were performed in SPSS 20.0 (IBM 2012).

All workers from unparasitized and parasitized colonies used in both behavioural and genomic experiments were dissected to confirm that they did not have developed ovaries nor yellow bodies (as sign of previous ovarian development), thus confirming they were not reproductively active (Cini 2014; Cini et al. 2013). During dissection workers were also checked for the presence of Strepsiptera endoparasites, which are known to be common in P. dominula (Hughes et al. 2004) and dramatically influence the wasp's behaviour and gene expression (Geffre et al. 2017). None of the workers included in the behavioural and transcriptomic analysis was found to be infected by Strepsiptera.

\section{Results}

None of the workers showed any signs of ovarian development in this study: all workers had threadlike ovarioles with no visible yolk nor yellow bodies.
Prediction 1: positions of workers differ in social networks of parasitized and unparasitized colonies

Colony size increased in both parasitized and unparasitized colonies during the experimental period (Wilcoxon test, $W=36, p=0.008, n=8$ ). At the end of the experiment colony size did not differ between parasitized and unparasitized colonies (Mann-Whitney test, $U=5.5, p=0.571, n=4$ ) (Supplementary Material S3).

Principal component analysis extracted three components with Eigenvalues greater than 1: PC1 (hereafter referred to as 'outgoing centrality') was mainly represented by outdegree, outcloseness, out eigenvector and betweenness, loadings range 0.604-0.852); PC2 (hereafter referred to as 'incoming centrality') was mainly represented by indegree, incloseness and in eigenvector, loadings range 0.717-0.808); finally PC3 was mainly correlated with clustering coefficient (loading 0.980). These three PCs performed well in condensing the social network metrics of individuals (variance explained 67.97\%) (Supplementary Material S3).

Social network metrics differed between workers on parasitized and unparasitized colonies, suggesting workers were responding at the behavioural level to the social parasite during the early stages of parasitism. Early evidence of response was apparent at day 1 after usurpation, at which time PC3 (mainly clustering coefficient) differed significantly with workers from parasitized colonies showing higher clustering centrality than workers from unparasitized colonies; this difference was not apparent by day 7 and day 14 (Table 3, Supplementary Material S4). Later evidence of response was apparent at day 14, where workers on parasitized colonies showed significantly greater outgoing centrality (PC1) and significantly higher incoming centrality (PC2) than workers from unparasitized colonies (Table 3, Supplementary Material S4); there was no significant difference before usurpation, at day 1 and at day 7. Colony of worker origin had no significant effect for any of the social network measures (Table 3, Supplementary Material S4) (Fig. 2). Overall, these data demonstrate that workers from parasitized colonies show significant modifications in their social behaviour within the dominance interaction network compared to workers from unparasitized colonies. In particular, they showed (at specific time-points) more interconnected networks and higher number of individuals performed and received dominance interactions.

\section{Prediction 2: worker brain gene expression differs in parasitized and unparasitized colonies}

Subtle differences in brain gene expression were detected between workers from parasitized and unparasitized colonies, suggesting that there are some neurogenomic changes among workers in response to usurpation by a social 
Table 3 Workers from parasitized colonies show differences in dominance network positions compared to workers from unparasitized colonies. Results of the node-level regression assessing the importance of parasitism status and colony of origin on centrality measures

\begin{tabular}{|c|c|c|c|c|c|}
\hline & & Coef & SE & $\mathrm{T}$ & c.Sig \\
\hline \multicolumn{6}{|c|}{ Pre-usurpation, $n=68$} \\
\hline \multirow[t]{3}{*}{$\mathrm{PC} 1$} & Intercept & -0.621 & 0.711 & -0.874 & 0.385 \\
\hline & colony & 0.005 & 0.013 & 0.353 & 0.725 \\
\hline & par.status & 0.397 & 0.339 & 1.170 & 0.246 \\
\hline \multirow[t]{3}{*}{ PC2 } & Intercept & 0.532 & 0.755 & 0.705 & 0.483 \\
\hline & colony & -0.009 & 0.014 & -0.654 & 0.515 \\
\hline & par.status & -0.173 & 0.360 & -0.482 & 0.632 \\
\hline \multirow[t]{3}{*}{ PC3 } & Intercept & 0.143 & 0.223 & 0.641 & 0.524 \\
\hline & colony & -0.005 & 0.004 & -1.194 & 0.237 \\
\hline & par.status & -0.158 & 0.106 & -1.483 & 0.143 \\
\hline \multicolumn{6}{|c|}{ Day 1 post-usurpation, $\mathrm{n}=55$} \\
\hline \multirow[t]{3}{*}{$\mathrm{PC} 1$} & Intercept & 0.531 & 0.392 & 1.354 & 0.182 \\
\hline & colony & -0.007 & 0.015 & -0.455 & 0.651 \\
\hline & par.status & -0.038 & 0.338 & -0.113 & 0.911 \\
\hline \multirow[t]{3}{*}{ PC2 } & Intercept & 0.842 & 0.321 & 2.620 & 0.011 \\
\hline & colony & -0.013 & 0.012 & -1.089 & 0.281 \\
\hline & par.status & 0.046 & 0.277 & 0.167 & 0.868 \\
\hline \multirow[t]{3}{*}{ PC3 } & Intercept & -0.336 & 0.468 & -0.719 & 0.475 \\
\hline & colony & 0.029 & 0.018 & 1.668 & 0.101 \\
\hline & par.status & 1391.000 & 0.403 & 3.448 & 0.001 \\
\hline \multicolumn{6}{|c|}{ Day 7 post-usurpation, $n=89$} \\
\hline \multirow[t]{3}{*}{ PC1 } & Intercept & -0.451 & 0.181 & -2.496 & 0.014 \\
\hline & colony & 0.012 & 0.007 & 1.801 & 0.075 \\
\hline & par.status & 0.044 & 0.159 & 0.280 & 0.780 \\
\hline \multirow[t]{3}{*}{$\mathrm{PC} 2$} & Intercept & -0.221 & 0.229 & -0.969 & 0.335 \\
\hline & colony & 0.008 & 0.009 & 0.893 & 0.375 \\
\hline & par.status & -0.148 & 0.201 & -0.735 & 0.464 \\
\hline \multirow[t]{3}{*}{ PC3 } & Intercept & -0.256 & 0.134 & -1.909 & 0.060 \\
\hline & colony & 0.004 & 0.005 & 0.697 & 0.487 \\
\hline & par.status & -0.100 & 0.118 & -0.847 & 0.400 \\
\hline \multicolumn{6}{|c|}{ Day 14 post-usurpation, $n=96$} \\
\hline \multirow[t]{3}{*}{$\mathrm{PC} 1$} & Intercept & -0.697 & 0.241 & -2.892 & 0.005 \\
\hline & colony & 0.012 & 0.009 & 1.311 & 0.193 \\
\hline & par.status & 1.037 & 0.215 & 4.820 & 0.000 \\
\hline \multirow[t]{3}{*}{$\mathrm{PC} 2$} & Intercept & -0.660 & 0.232 & -2.843 & 0.005 \\
\hline & colony & 0.007 & 0.009 & 0.845 & 0.400 \\
\hline & par.status & 0.715 & 0.207 & 3.447 & 0.001 \\
\hline \multirow[t]{3}{*}{ PC3 } & Intercept & -0.279 & 0.135 & -2.069 & 0.041 \\
\hline & colony & 0.001 & 0.005 & 0.103 & 0.919 \\
\hline & par.status & -0.047 & 0.120 & -0.390 & 0.697 \\
\hline
\end{tabular}

parasite. Parasitism had a significant effect on the expression level of the gene $I d g f 4$ (Wald chi-square $=4.341, d f=1$, $p=0.037)$ : workers from parasitized colonies showed lower Idgf4 expression than workers from unparasitized colonies (Fig. 3a, Supplementary Material S5). No effect of parasitism was found on the expression levels of the other four genes $(A R R$ : Wald chi-square $=0.058, d f=1, p=0.810$; HSP70: Wald chi-square $=0.849, d f=1, p=0.357 ;$ ApoLp III: Wald chi-square $=0.435, d f=1, p=0.530 ;$ MRJPI: Wald chi-square $=0.317, d f=1, p=0.574)$. Colony size had a significant effect on HSP70 expression level (HSP70: Wald chi-square $=4.507, d f=1, p=0.034$ ), with level of expression increasing with colony size, and on Idgf4 (Wald chi-square $=5.471, d f=1, p=0.019)$ with level of expression decreasing with colony size (Fig. 3b,c; Supplementary Material S5). Colony size had no effect on expression level of other genes $(A R R$ : Wald chi-square $=2.743$, $d f=1, p=0.098 ;$ ApoLp-III: Wald chi-square $=0.118$, $d f=1, p=0.731 ; M R J P 1:$ Wald chi-square $=1.521, d f=1$, $p=0.217$ ). The interaction between parasitism and colony size was significant only for the expression of $A R R$, whose expression increases with colony size in workers from unparasitized colonies but not in workers from parasitized colonies (ARR: Wald chi-square $=6.847, d f=1, p=0.009$; Fig. 3d; Supplementary Material S5). The interaction had no significant effect on the expression of the other four genes (Idgf4: Wald chi-square $=0.517, d f=1, p=0.472 ;$ HSP70: Wald chi-square $=0.559, d f=1, p=0.455$; ApoLp-III: Wald chi-square $=1.794, d f=1, p=0.180 ;$ MRJP1: Wald chisquare $=0.124, d f=1, p=0.725)$.

\section{Discussion}

In the strong coevolutionary arms race between social parasites and their hosts, hosts show a wide suite of defensive strategies, from avoidance strategies to reduce the chance of parasitism, to rebellion after parasitism has occurred (Brandt et al. 2005; Cervo 2006; Cini et al. 2019b; Grüter et al. 2018). The timing of host defence affects host fitness and a fast response to parasitism may help limit the costs incurred by the host due to the invasion. When parasitized by the social parasite $P$. sulcifer, $P$. dominula host workers show a covert reaction which consists of ovarian development, such that they are ready to reproduce if the opportunity arises (Cini et al. 2014). Here we highlighted that well before this physiological response is evident, significant modifications in worker social behaviour and brain gene expression occur. To our knowledge, this is the first evidence of early behavioural and neurogenomic responses by host workers towards the presence of an inquiline social parasite in a social insect.

Polistes workers from parasitized colonies were thought to not exhibit changes in their behaviours after being invaded by a social wasps, continuing to perform typical worker task, such as foraging and nest activities (Cervo 2006). Our timeseries dissection of social interactions, however, reveals subtle changes in their social behaviour from within a day of usurpation. On the day after the usurpation by the social parasite, workers showed significant changes in their clustering 

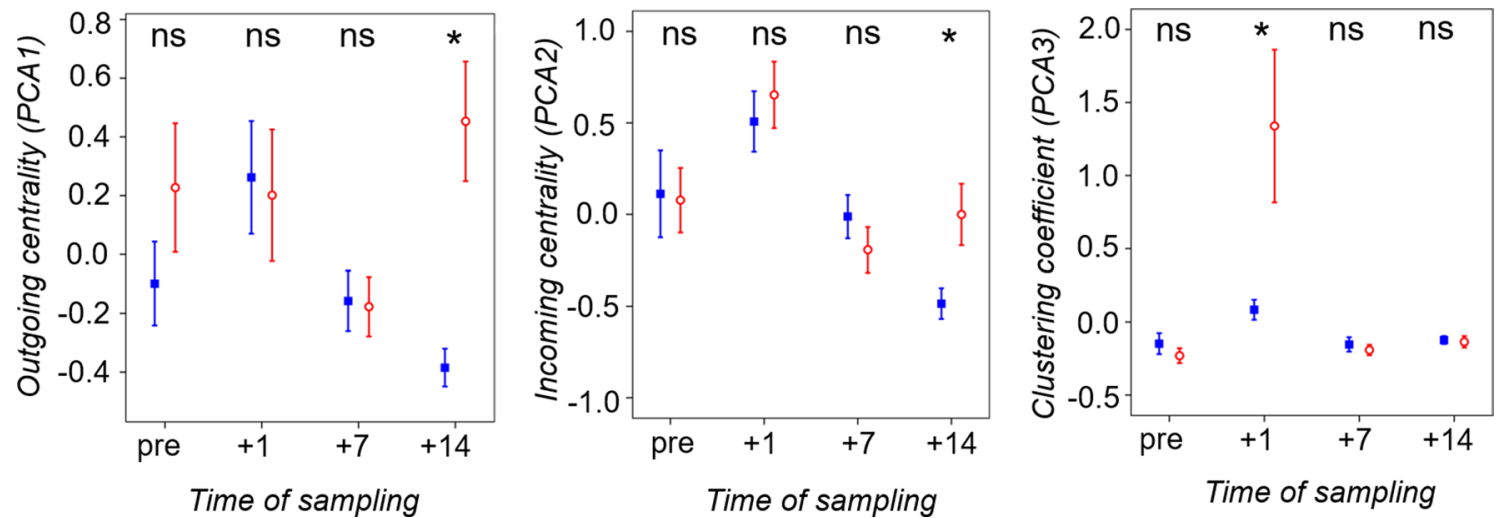

Fig. 2 Comparison in individual network centrality in workers from unparasitized colonies (squares) and parasitized (circles) before and across post-usurpation time (one, seven and fourteen days after usurpation). Mean and standard deviations are reported. An asterisk (*) indicates a significant difference, "ns" indicates no significant difference (see text and Table 3 for details)
Fig. 3 a Comparison in brain gene expression level for the target gene Idgf4 between workers from unparasitized (blue squares) and parasitized (red circles) colonies. Box plot reports the median value, the interquartile range and outliers are indicated with black circles. Bold font indicates significant differences for that target gene (see text and Table 3 for details); (b), (c) and (d) scatterplot of gene expression level vs colony size for workers from unparasitized (blue squares) and parasitized (red circles) colonies for the target genes HSP70, Idgf4 and ARR
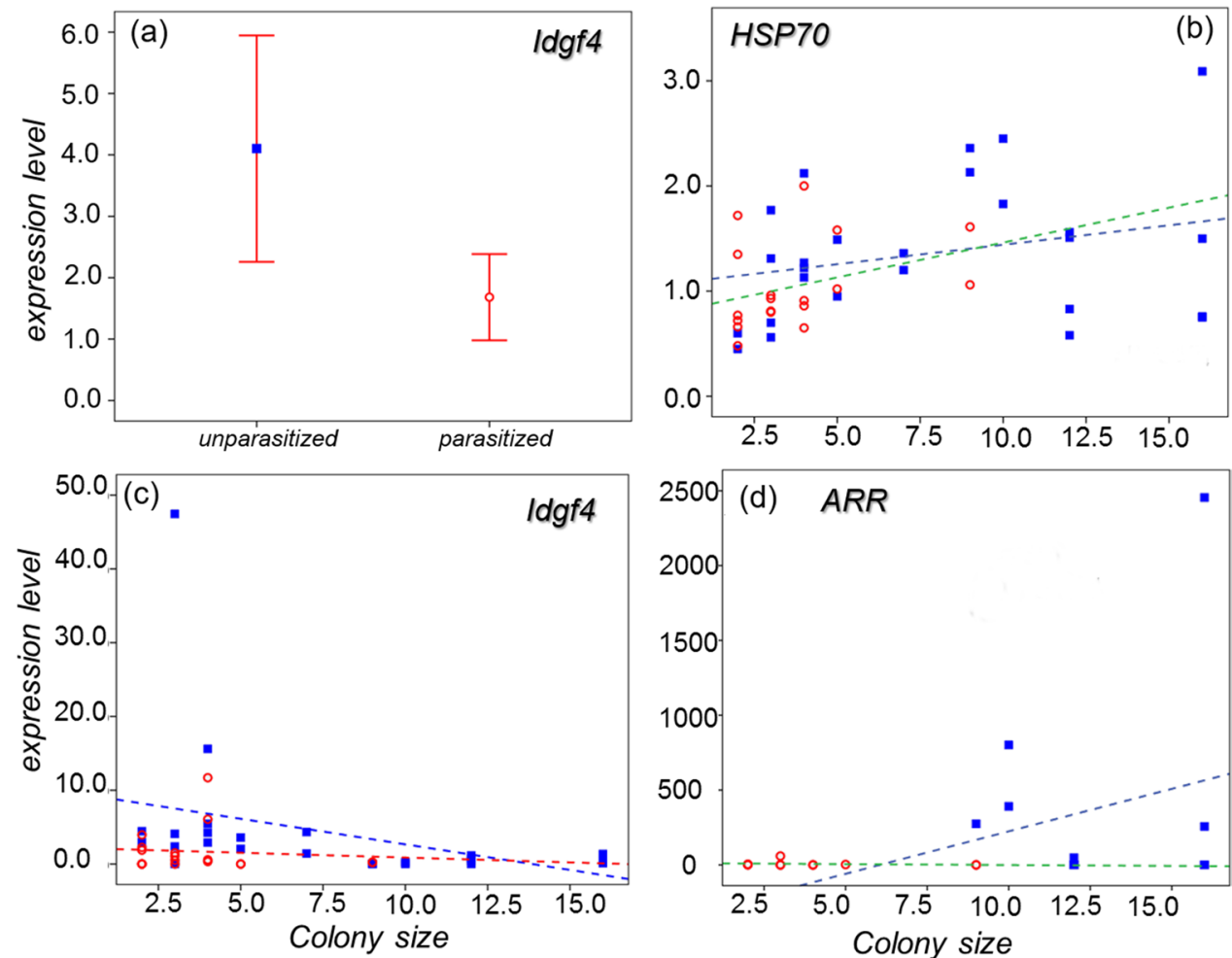

Unparasitized workers

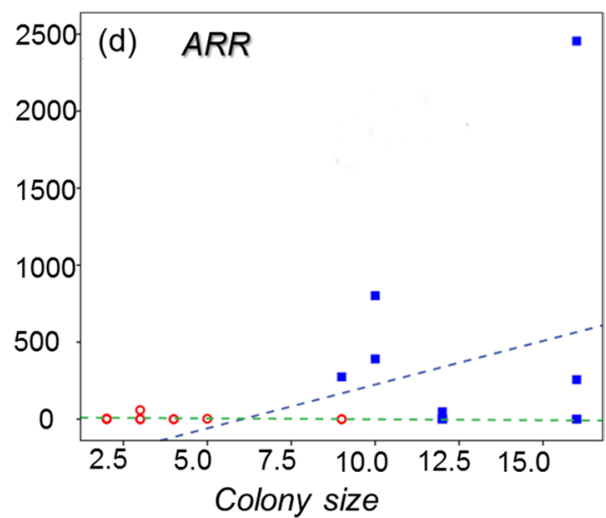

Parasitized workers coefficient: workers from usurped colonies exhibited denser (more interconnected) social networks than workers from unparasitized colonies. This could indicate that workers in parasitized colonies detect the intrusion of the social parasites from the outset of usurpation. The effect disappears by day 7. It is unclear at this stage whether the change in worker social networks on day 1 after usurpation is a response to the threat of the parasite specifically (i.e. they detect her as an alien intruder), or simply a response to a social perturbation that could equally arise within an unparasitized colony. In the former case, the response could be a reflection that the parasite has not yet become chemically integrated into the host colony: this is only achieved a few days after usurpation (Dapporto et al. 2004; Sledge et al. 2001; Turillazzi et al. 2000). The higher levels of connectedness amongst nest mates could be a form of information transfer and/or an attempt to reinforce the colony odour to defend against the successful take-over of the intruder. Conversely, the response on day 1 could simply reflect social disruption that occurs when a queen is lost and replaced, which may 
disrupt the dominance hierarchy (Jandt et al. 2014; Pardi 1948; Strassmann et al. 2004), resulting in an increase in RDI among colony members as they try to re-establish their dominance position in the new network. We lack social network analyses of the behavioural dynamics that take place after queen loss and replacement in Polistes; such studies would help determine whether the changes in centrality observed in parasitized workers is typical or not of queen succession. Equally, the parasite herself elicits high rates of RDI as she is particularly active during the first days of usurpation as she tries to dominate all individuals on the nest (Cervo 2006; Turillazzi et al. 1990): workers may be responding to general social perturbation within their worker ranks, rather than specific detection of the parasite per se. Future studies are required to directly assess whether such an increase in workers clustering is specific to the social disruption due to the parasite presence or if it is a generic response to any disturbance of colonial stability.

These arguments cannot, however, explain the result that workers from parasitized colonies also showed differences in their centrality within the colonial dominance network compared to unparasitized colonies two weeks after parasitism. We found that workers from parasitized colonies, by day 14 after usurpation, were significantly more central to the social network than workers from unparasitized colonies. In particular, workers from parasitized colonies showed a higher outgoing centrality (i.e. instigated more dominance interactions) and also a higher incoming centrality (i.e. received more dominance interactions) in the colonial dominance network compared to workers of unparasitized colonies. This finding is intriguing, as (a) dominance behaviour and reproduction are positively correlated in this species (Dapporto et al. 2010; Reeve et al. 1991), (b) a dominance hierarchy is established among workers, which regulate the access to reproduction in case the breeder is to be replaced (Jandt et al. 2014; Pardi 1948) and finally (c) more central individuals in dominance networks are typically the reproductive ones in wasps, as shown in Ropalidia species (Bhadra and Jordán 2013; Bhadra et al. 2009). Taking these factors into account, the finding that workers in parasitized colonies have higher outgoing and incoming centrality (i.e. perform and receive more dominance interactions) than workers from unparasitized colonies might suggest that the presence of the parasite leads to a social re-organization within the colony, with workers trying to escalate the dominance hierarchy to ensure future access to reproduction. The fact that workers in parasitized colonies showed higher levels of both performed and received dominance interactions might suggest that a clear dominance hierarchy was not yet achieved among workers. Our findings indeed parallel what has been found in Ropalidia wasps, where a reorganization of the dominance network, with increased centrality, has been shown after experimental breeder removal (Bhadra and Jordán 2013).
We also found some evidence that workers respond to parasitism from their brain gene expression. First, the gene $I d g f 4$ was downregulated in parasitized workers. Idgf4 is known to be involved in cuticle formation, organization of proteins in the chitin-matrix, and in adult moulting $(\mathrm{Gu}$ et al. 2019); this gene is putatively involved in responses to changes in the social environment in Polistes wasps (Cini et al. 2015). With respect to reproduction, in P. dominula $I d g f 4$ is upregulated in workers relative to queens in unparasitized colonies (Cini et al. 2015). A downregulation in the expression of Idgf4 in parasitized workers, therefore, may suggest that parasitized workers downregulate worker-biased genes, possibly instigating the switch from helper to reproductive. With respect to social environment, Idgf4 is upregulated in the social parasites when they shift from a solitary to social environment, upon taking over the colony and integrate into the host colony (Cini et al. 2015). The finding that workers downregulate Idgf4 expression in response to parasitism may indicate that, in the presence of the parasite, workers experience a change in the social environment. This is in accordance with the behavioural data, which shows behavioural changes in the colonial social network and signature of a social rearrangement.

The second gene whose expression pattern was influenced by parasite presence in our experiment was Arrestin; this has several roles of potential importance here. Firstly, the $A R R$ gene family is involved in visual and olfactory reception (Dolph et al. 1993; Merrill et al. 2002), it is linked to division of labour in bees, being upregulated in foragers and more defensive bees (Hunt et al. 2007; Sasagawa et al. 2003). In Polistes wasps, ARR was found to be associated with reproductive phenotype (Toth et al. 2014) and, specifically in the parasite species, was found responsive to changes in the social environment (Cini et al. 2015). While there was not an overall significant effect of parasitism on ARR expression, there was a significant crossover effect with colony size (i.e. a significant interaction). In workers from unparasitized colonies the expression of $A R R$ increased with colony size, this effect was not detected in workers from parasitized colonies. The increased expression of $A R R$ in bigger colonies (as found in workers from unparasitized colonies) thus makes sense under this perspective, as bigger colonies represent more crowded social environments, where more visual and chemical stimuli for social recognition are indeed to be processed. The finding that such a relationship does not hold for parasitized colonies is intriguing and suggests that, perhaps, social environment is disrupted in parasitized colonies such that workers do not (or cannot) respond to colony-size effects.

The neurogenomic responses of workers to social parasites reported here offer a tantalising first-look at the interactions between social parasites and their hosts, and support the behavioural responses we also report. However, we 
investigated only five candidate genes, and so our results represent a first step at understanding the response to parasitism. We hope these findings will encourage future work in this area, especially taking a genome-wide perspective (e.g. using RNAseq to sequence whole brain transcriptomes) along a temporal gradient from usurpation to the onset of evident, physiological modification in the parasite workers (6 weeks) (Cini et al. 2014). Matched analyses of genomic and behavioural changes over such a time course will also enable the critical question of whether the observed responses of parasitized workers are due to detection of the parasites (and thus a co-evolved response), or simply a response to conspecific colony perturbations. Genomic analyses will help reveal the nature of any co-evolved mechanism, e.g. in olfactory proteins, such as chemosensory and odorant binding molecules (Calvello et al. 2005; Pelosi et al. 2005, 2006, 2014; Zhu et al. 2019, but also see Xiao et al. 2019 for recent findings on the controversial role of odorant binding proteins) that may allow the host workers to detect the parasite.

The idea that workers might be able detect the social parasite would then raise interesting and important questions about the cues that workers use to do so. Workers could recognize the presence of the parasite by two main sensory modalities: smell, by detecting cues associated with the parasite itself or with its brood (eggs, larvae and pupae) (Cervo et al. 2008; Cini et al. 2014; Lorenzi 2006) or sight (Cini et al. 2019), by recognising the facial pattern of the parasite, a major point of difference in parasite and host morphology (Cervo et al. 2015; Cini et al. 2015; Ortolani et al. 2010). The timing of workers response (day 1 and day 14) suggests that visual (facial recognition) and chemical cues (recognition of the odour of the adult) might be the cues informing workers of parasite presence. Indeed, facial pattern is evidently a static cue, which does not change with time and can be assessed from the beginning of the host-parasite interaction [and indeed it plays a role in parasite infection, reducing host aggressiveness toward social parasites (Ortolani et al. 2010)]. Similarly, the odour of the social parasite is expected to play a role in the very first days after the usurpation since $P$. sulcifer is able to chemically integrate into the host colony within 3-4 days after usurpation. Therefore, we would expect a behavioural reaction to occur rapidly after usurpation if parasite odour was the detection cue. The idea that parasite brood odour is the key cue is not supported by our findings. Indeed, eggs and larvae of the social parasites are unlikely to allow detection, as the odour of parasite eggs is very similar to that of host eggs, and larvae escape detection by bearing a meaningless odour (Cervo et al. 2008; Dani et al. 2004). The remaining developmental stages of parasite brood, i.e. pupae and newly emerged brood, will not be present in the colony until, on average, 15 and 25 days (Cervo et al. 2004). This suggests that chemical cues from parasite brood are unlikely to allow detection of the parasite, leaving open the possibility that social parasites are detected because of some visual or odorant cue of the social parasite itself.

Overall, our study shows that host workers respond at the behavioural and brain transcriptomic level to social parasitism, from the outset. We cannot yet determine which of these responses are a result of a co-evolutionary adaptation by hosts to minimise costs of the parasite, or if they are a typical response to within-colony perturbations that any unparasitized colony could have experienced. A co-evolved response should carry strong selection pressures because an immediate and overt reaction to the social parasite would be risky for the workers. Indeed, $P$. sulcifer is bigger than the host, has a greater fighting ability and can easily defeat up to 11 workers (Cervo and Turillazzi 1996; Cini et al. 2011). A subtle response, as observed here, therefore may be adaptive. Our results provide the first evidence of early behavioural and neurogenomic responses of host workers toward the presence of an inquiline social parasite in a social insect, highlighting the importance of considering the timing of defensive responses when analysing the ongoing co-evolutionary arms race between hosts and their social parasites.

Acknowledgements We wish to thank George Busby for help in geneexpression analysis, Federico Cappa and Rachele Nieri for help with wasp rearing and behavioural observations. Funds were provided by University of Florence to RC, by the Marie Sklodowska-Curie Action, grant no. 706208 SocParPhenoEvol, to A.C. and by the Natural Environment Research Council (NERC; grant ref NE/G000638/1) to SS.

Open Access This article is licensed under a Creative Commons Attribution 4.0 International License, which permits use, sharing, adaptation, distribution and reproduction in any medium or format, as long as you give appropriate credit to the original author(s) and the source, provide a link to the Creative Commons licence, and indicate if changes were made. The images or other third party material in this article are included in the article's Creative Commons licence, unless indicated otherwise in a credit line to the material. If material is not included in the article's Creative Commons licence and your intended use is not permitted by statutory regulation or exceeds the permitted use, you will need to obtain permission directly from the copyright holder. To view a copy of this licence, visit http://creativecommons.org/licenses/by/4.0/.

\section{References}

Achenbach A, Foitzik S (2009) First evidence for slave rebellion: enslaved ant workers systematically kill the brood of their social parasite Protomognathus americanus. Evolution (N Y) 63:10681075. https://doi.org/10.1111/j.1558-5646.2009.00591.x

Bhadra A, Jordán F (2013) Cryptic successors unrevealed even by network analysis: a comparative study of two paper wasp species. Netw Biol 3:54-66. https://doi.org/10.0000/issn-2220-8879-netwo rkbiology-2013-v3-0005

Bhadra A, Jordán F, Sumana A et al (2009) A comparative social network analysis of wasp colonies and classrooms: linking network structure to functioning. Ecol Complex 6:48-55. https://doi. org/10.1016/j.ecocom.2008.10.004 
Borgatti SP, Everett MG, Freeman LC (2002) UCINET 6 For Windows: software for social network analysis, Analytic Technologies, Harvard, MA. Anal Technol

Brandt M, Foitzik S, Fischer-Blass B, Heinze J (2005) The coevolutionary dynamics of obligate ant social parasite systems - between prudence and antagonism. Biol Rev Camb Philos Soc 80:251267. https://doi.org/10.1017/S1464793104006669

Bruschini C, Cervo R, Turillazzi S (2010) Pheromones in social wasps. Academic Press, Cambridge

Burton P, Gurrin L, Sly P (1998) Extending the simple linear regression model to account for correlated responses: an introduction to generalized estimating equations and multi-level mixed modelling. Stat Med 17:1261-1291. https://doi.org/10.1002/(SICI)10970258(19980615)17:11\%3c1261:AID-SIM846\%3e3.0.CO;2-Z

Calvello M, Brandazza A, Navarrini A et al (2005) Expression of odorant-binding proteins and chemosensory proteins in some Hymenoptera. Insect Biochem Mol Biol 35:297-307

Cervo R (2006) Polistes wasps and their social parasites: an overview. Ann Zool Fenn 43:531-549. https://doi.org/10.2307/23736760

Cervo R, Cini A, Turillazzi S (2015) Visual recognition in social wasps. In: Aquiloni L, Tricarico E (eds) Social recognition in invertebrates. Springer, Cham, pp 125-145

Cervo R, Dani FR (1996) Social parasitism and its evolution in Polistes. [W:] Natural history and evolution of paper-wasps. Turillazzi S., West-Eberhard MJ (eds.)

Cervo R, Dani FR, Cotoneschi C et al (2008) Why are larvae of the social parasite wasp Polistes sulcifer not removed from the host nest? Behav Ecol Sociobiol 62:1319-1331. https://doi. org/10.1007/s00265-008-0560-1

Cervo R, Macinai V, Dechigi F, Turillazzi S (2004) Fast growth of immature brood in a social parasite wasp: a convergent evolution between avian and insect cuckoos. Am Nat 164:814-820. https:// doi.org/10.1086/425987

Cervo R, Turillazzi S (1996) Host nest preference and nest choice in the cuckoo paper wasp Polistes sulcifer (Hymenoptera: Vespidae). J Insect Behav 9:297-306. https://doi.org/10.1007/BF02213872

Cini A (2014) Reliability of yellow bodies as indexes of egg laying activity in the primitively eusocial wasp Polistes dominula. Redia 97:183-188

Cini A, Bruschini C, Poggi L, Cervo R (2011) Fight or fool? Physical strength, instead of sensory deception, matters in host nest invasion by a wasp social parasite. Anim Behav 81:1139-1145. https ://doi.org/10.1016/j.anbehav.2011.02.017

Cini A, Bruschini C, Signorotti L et al (2011) The chemical basis of host nest detection and chemical integration in a cuckoo paper wasp. J Exp Biol 214:3698-3703. https://doi.org/10.1242/ jeb.059519

Cini A, Cappa F, Pepiciello I et al (2019) Sight in a clique, scent in society: plasticity in the use of nestmate recognition cues along colony development in the social wasp Polistes dominula. Front Ecol Evol 7:444. https://doi.org/10.3389/fevo.2019.00444

Cini A, Dapporto L (2009) Autumnal helpers of Polistes dominulus represent a distinct behavioural phenotype. Ann Zool Fennici 46:423-430. https://doi.org/10.5735/086.046.0603

Cini A, Meconcelli S, Cervo R (2013) Ovarian indexes as indicators of reproductive investment and egg-laying activity in social insects: a comparison among methods. Insectes Soc 60:393-402. https:// doi.org/10.1007/s00040-013-0305-7

Cini A, Nieri R, Dapporto L et al (2014) Almost royal: Incomplete suppression of host worker ovarian development by a social parasite wasp. Behav Ecol Sociobiol 68:467-475. https://doi.org/10.1007/ s00265-013-1661-z

Cini A, Ortolani I, Zechini L, Cervo R (2015) Facial markings in the social cuckoo wasp Polistes sulcifer: No support for the visual deception and the assessment hypotheses. Behav Processes 111:19-24

Cini A, Patalano S, Segonds-Pichon A et al (2015) Social parasitism and the molecular basis of phenotypic evolution. Front Genet 6:32. https://doi.org/10.3389/fgene.2015.00032

Cini A, Sumner S, Cervo R (2019b) Inquiline social parasites as tools to unlock the secrets of insect sociality. Philos. Trans. R. Soc. B Biol. Sci. 374

Dani FR, Giovannotti M, Cervo R, Turillazzi S (2004) Esiste integrazione chimica fra la prole del parassita sociale Polistes sulcifer e quella del suo ospite $P$. dominulus (Hymenoptera: Vespidae). In: XIX Congresso Nazionale Italiano di Entomologia, Catania, giugno. pp 377-380. https://flore.unifi.it/handle/2158/67569 1\#.XqoFJ6j7SUk

Dapporto L, Bruschini C, Cervo R, Petrocelli I, Turillazzi S (2010) Hydrocarbon rank signatures correlate with differential oophagy and dominance behaviour in Polistes dominulus foundresses. $\mathrm{J}$ Exp Biol 213(3):453-458. https://doi.org/10.1242/jeb.032938

Dapporto L, Cervo R, Sledge MF, Turillazzi S (2004) Rank integration in dominance hierarchies of host colonies by the paper wasp social parasite Polistes sulcifer (Hymenoptera, Vespidae). J Insect Physiol 50:217-223. https://doi.org/10.1016/j.jinsphys.2003.11.012

Dawkins R, Krebs JR (1979) Arms races between and within species. Proc R Soc London Biol Sci 205:489-511. https://doi. org/10.1098/rspb.1979.0081

Dolph PJ, Ranganathan R, Colley NJ et al (1993) Arrestin function in inactivation of $\mathrm{G}$ protein-coupled receptor rhodopsin in vivo. Science (80- ) 260:1910-1916

Drapeau MD, Albert S, Kucharski R et al (2006) Evolution of the yellow/major royal jelly protein family and the emergence of social behavior in honey bees. Genome Res 16(11):1385-1394. https:// doi.org/10.1101/gr.5012006

Ferguson LC, Green J, Surridge A, Jiggins CD (2011) Evolution of the insect yellow gene family. Mol Biol Evol. 28(1):257-272. https:// doi.org/10.1093/molbev/msq192

Ferreira PG, Patalano S, Chauhan R, et al (2013) Transcriptome analyses of primitively eusocial wasps reveal novel insights into the evolution of sociality and the origin of alternative phenotypes. Genome Biol. https://doi.org/10.1186/gb-2013-14-2-r20

Geffre AC, Liu R, Manfredini F et al (2017) Transcriptomics of an extended phenotype: parasite manipulation of wasp social behaviour shifts expression of caste-related genes. Proc R Soc B Biol Sci 284:20170029. https://doi.org/10.1098/rspb.2017.0029

Grüter C, Jongepier E, Foitzik S (2018) Insect societies fight back: the evolution of defensive traits against social parasites. Phil Trans R Soc B 373:20170200. https://doi.org/10.1098/rstb.2017.0200

$\mathrm{Gu}$ X, Li Z, Su Y et al (2019) Imaginal disc growth factor 4 regulates development and temperature adaptation in Bactrocera dorsalis. Sci Rep 9:1-11

Heinze J (2008) Hierarchy length in orphaned colonies of the ant Temnothorax nylanderi. Naturwissenschaften 95:757-760. https://doi. org/10.1007/s00114-008-0375-y

Hölldobler B, Wilson EO (1990) The ants. Harvard University Press, Cambridge

Hubbard AE, Ahern J, Fleischer NL et al (2010) To GEE or not to GEE: comparing population average and mixed models for estimating the associations between neighborhood risk factors and health. Epidemiology 21:467-474

Hughes DP, Kathirithamby J, Beani L (2004) Prevalence of the parasite Strepsiptera in adult Polistes wasps: field collections and literature overview. Ethol Ecol Evol 16:363-375

Hunt GJ (2007) Flight and fight: a comparative view of the neurophysiology and genetics of honey bee defensive behavior. J Insect Physiol 53:399-410. https://doi.org/10.1016/j.jinsphys.2007.01.010 
Hunt GJ, Amdam GV, Schlipalius D et al (2007) Behavioral genomics of honeybee foraging and nest defense. Naturwissenschaften 94:247-267. https://doi.org/10.1007/s00114-006-0183-1

Hunt JH, Wolschin F, Henshaw MT, et al (2010) Differential gene expression and protein abundance evince ontogenetic bias toward castes in a primitively eusocial wasp. PLoS One 5. https://doi. org/10.1371/journal.pone.0010674

IBM Corp. (2011) IBM SPSS Statistics for Windows, Version 20.0. IBM Corp, Armonk, NY

Jandt JM, Tibbetts EA, Toth AL (2014) Polistes paper wasps: a model genus for the study of social dominance hierarchies. Insectes Soc 61:11-27. https://doi.org/10.1007/s00040-013-0328-0

King AM, MacRae TH (2015) Insect heat shock proteins during stress and diapause. Annu Rev Entomol 60:59-75. https://doi. org/10.1146/annurev-ento-011613-162107

Krause J, Lusseau D, James R (2009) Animal social networks: an introduction. Behav Ecol Sociobiol 63:967-973

Lhomme P, Hines HM (2018) Ecology and evolution of cuckoo bumble bees. Ann Entomol Soc Am. https://doi.org/10.1093/aesa/say031

Lorenzi MC (2006) The result of an arms race: the chemical strategies of Polistes social parasites. Ann Zool Fenn 43:550-563

Lorenzi MC, Azzani L, Bagnéres A-G et al (2014) Evolutionary consequences of deception: complexity and informational content of colony signature are favored by social parasitism. Curr Zool 60:137-148. https://doi.org/10.1093/czoolo/60.1.137

Lowe RM, Ward SA, Crozier RH (2002) The evolution of parasites from their hosts: intra- and interspecific parasitism and Emery's rule. Proc R Soc B Biol Sci 269:1301-1305. https://doi. org/10.1098/rspb.2002.2008

Manfredini F, Brown MJFF, Toth AL (2018) Candidate genes for cooperation and aggression in the social wasp Polistes dominula. J Comp Physiol A 204:449-463. https://doi.org/10.1007/s0035 9-018-1252-6

Martin SJ, Helanterä H, Drijfhout FP et al (2011) Is parasite pressure a driver of chemical cue diversity in ants? Proc Biol Sci 278:496-503. https://doi.org/10.1098/rspb.2010.1047

Merrill CE, Riesgo-Escovar J, Pitts RJ et al (2002) Visual arrestins in olfactory pathways of Drosophila and the malaria vector mosquito Anopheles gambiae. Proc Natl Acad Sci 99:1633-1638

Monnin T, Cini A, Lecat V et al (2009) No actual conflict over colony inheritance despite high potential conflict in the social wasp Polistes dominulus. Proc Biol Sci 276:1593-1601. https://doi. org/10.1098/rspb.2008.1739

Monnin T, Ratnieks FLW, Brandão CRF (2003) Reproductive conflict in animal societies: Hierarchy length increases with colony size in queenless ponerine ants. Behav Ecol Sociobiol 54:71-79. https ://doi.org/10.1007/s00265-003-0600-9

Naug D (2008) Structure of the social network and its influence on transmission dynamics in a honeybee colony. Behav Ecol Sociobiol 62:1719-1725. https://doi.org/10.1007/s00265-008-0600-x

Ortolani I, Cervo R (2010) Intra-specific body size variation in Polistes paper wasps as a response to social parasite pressure. Ecol Entomol 35:352-359. https://doi.org/10.1111/j.1365-2311.2010.01187 . $\mathrm{x}$

Ortolani I, Turillazzi S, Cervo R (2008) Spring usurpation restlessness: A wasp social parasite adapts its seasonal activity to the host cycle. Ethology 114:782-788. https://doi.org/10.111 1/j.1439-0310.2008.01525.x

Ortolani I, Zechini L, Turillazzi S, Cervo R (2010) Recognition of a paper wasp social parasite by its host: evidence for a visual signal reducing host aggressiveness. Anim Behav 80:683-688. https:// doi.org/10.1016/j.anbehav.2010.07.003

Overall JE, Tonidandel S (2004) Robustness of generalized estimating equation (GEE) tests of significance against misspecification of the error structure model. Biometrical J 46:203-213. https://doi. org/10.1002/bimj.200210017
Pan W, Wall MM (2002) Small-sample adjustments in using the sandwich variance estimator in generalized estimating equations. Stat Med 21:1429-1441

Pardi L (1948) Dominance order in Polistes wasps. Physiol Zool 21:113. https://doi.org/10.1086/physzool.21.1.30151976

Pelosi P, Calvello M, Ban L (2005) Diversity of odorant-binding proteins and chemosensory proteins in insects. Chem Senses 30:i291-i292

Pelosi P, Iovinella I, Felicioli A, Dani FR (2014) Soluble proteins of chemical communication: an overview across arthropods. Front Physiol 5:320

Pelosi P, Zhou J-J, Ban LP, Calvello M (2006) Soluble proteins in insect chemical communication. Cell Mol Life Sci C 63:1658-1676

Pinter-Wollman N, Hobson EA, Smith JE et al (2014) The dynamics of animal social networks: Analytical, conceptual, and theoretical advances. Behav Ecol 25:242-255. https://doi.org/10.1093/ beheco/art047

Reed HC, Akre RD (1983) Colony behavior of the obligate social parasite Vespula austriaca (Panzer) (Hymenoptera: Vespidae). Insectes Soc 30:259-273. https://doi.org/10.1007/BF02223984

Reeve HK (1991) Polistes. In: Ross K, Matthews R (eds) The social biology of wasp. Cornell University Press, Ithaca, NY, pp 99-148

Robinson GE, Grozinger CM, Whitfield CW (2005) Sociogenomics: social life in molecular terms. Nat Rev Genet 6:257-270. https:// doi.org/10.1038/nrg 1575

Rubenstein DR, Hofmann HA (2015) Proximate pathways underlying social behavior Curr Opinion Behav. Sciences 6:154-159. https ://doi.org/10.1016/j.cobeha.2015.11.007

Sasagawa H, Narita R, Kitagawa Y, Kadowaki T (2003) The expression of genes encoding visual components is regulated by a circadian clock, light environment and age in the honeybee (Apis mellifera). Eur J Neurosci 17:963-970. https://doi.org/10.104 6/j.1460-9568.2003.02528.x

Sledge MF, Dani FR, Cervo R et al (2001) Recognition of social parasites as nest-mates: Adoption of colony-specific host cuticular odours by the paper wasp parasite Polistes sulcifer. Proc R Soc B Biol Sci 268:2253-2260. https://doi.org/10.1098/rspb.2001.1799

Smith JA, Chenoweth LB, Tierney SM, Schwarz MP (2013) Repeated origins of social parasitism in allodapine bees indicate that the weak form of Emery's rule is widespread, yet sympatric speciation remains highly problematic. Biol J Linn Soc 109:320-331. https://doi.org/10.1111/bij.12043

Strassmann JE, Fortunato A, Cervo R et al (2004) The cost of queen loss in the social wasp Polistes dominulus (Hymenoptera: Vespidae). J Kansas Entomol Soc 77:343-355. https://doi. org/10.2317/E-15.1

Sumner S, Pereboom JM, Jordan WC (2006) Differential gene expression and phenotypic plasticity in behavioural castes of the primitively eusocial wasp, Polistes canadensis. Proc R Soc B Biol Sci 273:19-26. https://doi.org/10.1098/rspb.2005.3291

Toth AL, Tooker JF, Radhakrishnan S et al (2014) Shared genes related to aggression, rather than chemical communication, are associated with reproductive dominance in paper wasps (Polistes metricus). BMC Genomics 15:75. https://doi.org/10.1186/1471-2164-15-75

Toth AL, Varala K, Newman TC et al (2007) Wasp gene expression supports an evolutionary link between maternal behavior and eusociality. Science (80- ) 318:441-444. https://doi.org/10.1126/ science. 1146647

Turillazzi S, Cervo R, Cavallari I (1990) Invasion of the Nest of Polistes dominulus by the Social Parasite Sulcopolistes sulcifer (Hymenoptera, Vespidae). Ethology 84:47-59. https://doi. org/10.1111/j.1439-0310.1990.tb00784.x

Turillazzi S, Sledge MF, Dani FR et al (2000) Social hackers: Integration in the host chemical recognition system by a paper wasp social parasite. Naturwissenschaften 87:172-176. https://doi. org/10.1007/s001140050697 
Valente TW, Coronges K, Lakon C, Costenbader E (2008) How correlated are network centrality measures? Connect (Tor) 28:16

Weers PMM, Ryan RO (2006) Apolipophorin III: role model apolipoprotein. Insect Biochem Mol Biol 36(4):231-240

Wey T, Blumstein DT, Shen W et al (2008) Social network analysis of animal behaviour: a promising tool for the study of sociality. Anim Behav 75:333-344. https://doi.org/10.1016/j.anbehav.2007.06.020

Wilson EO (1971) The Insect Societies. Harvard Univ Press, Cambridge, Mass
Xiao S, Sun JS, Carlson JR (2019) Robust olfactory responses in the absence of odorant binding proteins. Elife. https://doi.org/10.7554/ eLife. 51040

Zhu J, Iovinella I, Dani FR et al (2019) Chemosensory proteins: a versatile binding family. Olfactory concepts of insect controlalternative to insecticides. Springer, Berlin, pp 147-169 